\title{
BOOSTING APPROACH TO EARLY BANKRUPTCY PREDICTION FROM MULTIPLE-YEAR FINANCIAL STATEMENTS
}

\author{
Yuta Takata a , Tadaaki Hosaka ${ }^{b}$, Hiroshi Ohnuma ${ }^{c}$ \\ abc Tokyo University of Science, Tokyo, Japan \\ Corresponding email: 8615604@ed.tus.ac.jp
}

\begin{abstract}
Predicting business failure is primarily composed of two processes: selecting financial indicators and constructing a prediction model. Many traditional studies separate these two tasks and do not guarantee the entire process optimization. We have, thus far, used the AdaBoost algorithm to integrate a selection of indicators and a construction of a prediction model within a single coherent framework. However, this model is only valid for one-year of future predictions. The study in this article attempts to extend our method to predict business failures earlier by using time-series financial data. We define several types of financial ratios which are generated from two arbitrary items in the time-series balance sheets, profit-and-loss statements and cash flow statements as candidates of indicators. Further, our boosting-based method selects an effective combination of financial ratios and derives a discrimination function, which can predict companies' bankruptcy within a few years. Evaluation experiments indicate that our method can produce predictions one year ahead of bankruptcy with an identification rate of $82.9 \%$. Moreover, the following three ratios are demonstrated as the most powerful indicators: 1) Retained earnings (present value)/ Current assets (present value);2) Valuation, translation adjustments, and others (the difference between the most recent two years) / Capital stock(present value); and 3) Loss on disposal, valuation of other assets (sum over the most recent two years) / Capital stock (sum over the most recent two years).
\end{abstract}

Keywords: Business Failure, AdaBoost, Time-series Financial Data.

\section{Introduction}

It is important to estimate a company's future from its current financial and management condition to generate profit in trading securities. One of these directions is represented through the prediction of corporate bankruptcies and many bankruptcy predictions have been reported from statistical perspectives.

Crucial factors in bankruptcy prediction studies include the selection of financial indicators to use in the analysis and construction of a predictive model. Many previous bankruptcy prediction studies manually establish their financial indicators based on accounting perspectives and on the knowledge from past studies. This type of works emphasizes the evaluation of accuracy in the proposed prediction models, for which such statistical techniques as pattern recognition and machine learning were frequently utilized(Altman, 1968; Shin, Lee \& Kim, 2005; Ramakrishnan, Mirzaei \& Bekri, 2015). These studies do not necessarily guarantee optimization between the manually chosen financial indicators and a manually adopted prediction model. On the other hand, some studies have selected financial indicators using statistical methods, such as decision trees(Shirata, 1999; Shirata, 2003). Many of these studies apply classifiers in a later stage, such as the linear discriminant analysis. As these two processes are separately implemented, the selected financial indicators are not necessarily appropriate for the prediction models. 
We have, thus far, utilized AdaBoost (Takata, Hosaka \& Ohnuma, 2015) and its advanced version, RealAdaBoost (Hosaka \& Takata, 2016)to select financial indicators and construct a prediction model within a single framework. AdaBoost proposed by Freund and Schapire(1997)and RealAdaBoost proposed by Schapire and Singer (1999) have been occasionally used for feature vector selection in the machine learning field, and our past studies have regarded these techniques as applicable to financial analysis. AdaBoost iteratively chooses a financial indicator from a mass of candidates, and the weight of learning samples that are mistakenly classified by the selected indicator increases in the next iteration step, naturally extracting a combination of complimentary indicators. This algorithm also provides a method to construct the discriminant function (prediction model) using the selected indicators in a common single framework. However, our past studies handled learning samples collected within one year before bankruptcy; therefore, the derived discriminant function is effective for only one-year future predictions.

This article extends our method for earlier bankruptcy prediction. We utilize time-series financial data (balance sheets, profit-and-loss statements, and cash flow statements) and define several types of financial ratios that correspond to the sum and difference over two fiscal years as candidates of indicators. Our boosting-based approach selects a combination of financial ratios and derives the discriminant function effective to predict companies' bankruptcy in a few years in the future.

\section{Literature on Business Failure Predictions}

Beaver (1967) first provided a bankruptcy prediction based on a statistical approach. He used subjective insight to collect 30 cash flow-based financial ratios, which past studies often utilized to generate favorable results, and investigated the discrimination capabilities of each ratio.

Altman (1968) first attempted to simultaneously handle multiple financial ratios. This study used financial data from 33 failed manufacturers, which filed for bankruptcy during 19461965, and that from 33 continuing companies of approximately the same scale. He also manually selected 22 financial indicators that can be interpreted from the liquidity, profitability, leverage, solvency, and turnover rate perspectives. The classification performances of various combinations among these ratios were examined through a linear discriminant analysis, and five financial ratios were ultimately chosen as effective predictors: 1) Working capital / Total capital; 2) Retained earnings / Total assets; 3) Earnings before interest and taxes / Total assets;4)Market value of equity interests / Total assets; and 5) Amount of sales / Total assets.

Statistical methods were also later introduced in the financial indicator selection process. For instance, Edmister (1972) selected financial ratios by using the sequential variable selection method,and Sung (1999)and Shirata (1999; 2003)used the decision tree algorithm to select financial indicators effective in bankruptcy predictions. Shirata's (2003)prediction model, called SAF (Simple Analysis of Failure) 2002, eventually selected four indicators: 1) Retained earnings / Total assets; 2) Net income before taxes / Total assets; 3) Inventory $(\times 12)$ / Amount of sales; and 4) Interest expenses / Amount of sales. This model was widely used as a benchmark in subsequent research.

Non-linear statistical methods have often been utilized to improve prediction accuracy. Examples include Ohlson's(1980) research, which used the logistic regressions; Altman et al.'s (1994) work, which useda neural network; or Shin et al.'s(2005) study, which applied the support vector machine to bankruptcy prediction. AdaBoost, which our study will also 
use, appeared in literature (Ramakrishnan, Mirzaei \& Bekri, 2015) not to select financial indicators, but to construct a prediction model.

The aforementioned studies separately implement the two crucial processes of indicator selection and model construction. Therefore, we believe that no guarantee exists that the final discrimination accuracy is optimized throughout the entire process. Thus far, we have utilized AdaBoost (Takata, Hosaka \& Ohnuma, 2015) to solve this problem, as well as an extended version, RealAdaBoost (Hosaka \& Takata, 2016). These two methods can both select financial indicators and construct a prediction model within a single common coherent framework.

Some research has utilized time-series financial data to investigate early predictions' accuracy. Although Altman's (1968) aforementioned model could identify bankruptcy within one year, with $95 \%$ accuracy, the discrimination rate decreased to $72 \%$ for two years ahead of bankruptcy and $48 \%$ for three years ahead of bankruptcy. Shirata(1999) examined her model's applicability to time-series data, and noted that many bankrupt companies exhibited nearly constant values for the discriminant function for a fairly long time (e.g., ten years), which indicates that a bankruptcy's precise timing is difficult to predict. It seems that earlier predictions of more than three years generally pose highly difficult problems.

This study aims to construct a highly accurate prediction model that can identify companies that will go bankrupt in a few years by applying time-series financial statements to our boosting approach.

\section{Methodology}

\subsection{Dataset}

Japan has no legal definition for bankruptcy; however, it is typically regarded as the "incapability of settling liabilities due." Failed companies, as the subjects of this study, are defined based on this understanding as companies that delisted their shares due to financial difficulties. We first collect all companies delisted from the Tokyo Stock Exchange, the Osaka Stock Exchange, the former NASDAQ Japan Standard, the former Hercules Standard, and the former JASDAQ in or after 2002. Additionally, this list was confined to 94 de facto bankrupt companies which have been delisted by their negative net worth; suspension of bank transactions; filing for bankruptcy, revitalization, or reorganization; and the termination of business activities, except for mergers. Financial institutions, including banks, are not covered in the study due to the non-availability of their financial information. On the other hand, continuing companies in this study include all companies listed on the Tokyo Stock Exchange as of June 2016, or a total of 2,287 companies.

The consolidated balance sheets, profit-and-loss statements, and cashflow statements for the 94 failed companies and 2,287 continuing companies are obtained from the Nikkei NEEDS Financial QUEST database. We collect failed companies' statements for the four fiscal years before their delisting, and the latest includes data within one year of the delisting. Regarding continuing companies, we handle the data reported within one year prior to June 2016 as the most recent, and acquire financial statements for four fiscal years; namely, the data period ranges from 2013 to 2016. Each one-year dataset for one company consists of 182 items in the balance sheet, 88 items in the profit-and-loss statement, and 96 items in the cash flow statement. 


\subsection{Data Pre-processing}

Financial statements include many missing values because:1) specific items do not exist depending on the industry, 2) zero values cannot be reported, and3) sub-entries in the main item cannot be reported. This study addresses these missing values using the following two methods:

a)In Japan, the accounting standards for the net assets section significantly changed in 2006, which caused some missing data, primarily for capital stock-related items. Considering the corresponding relationship between the new and old standards, the missing values are appropriately complemented by other financial items.

b) Regardless of the renewal of accounting standards, missing values are complemented for financial items that can be calculated or estimated from other items.

After the above manipulation, we checked for each financial item if more than half of the 94 failed companies or 2,287 continuing companies have zero values or missing values. This task is performed on each year's data, and financial concerns in any years are removed from every year's data. Subsequently, 52 financial items out of 366 financial items remain, and the remaining missing values in those financial items were replaced by 0 .

\subsection{Generation of candidate financial ratios}

The complete data after pre-processing is used to generate all conceivable financial ratios. AdaBoost eventually extracts the best combination of ratios effective for bankruptcy prediction. This study considers two types of financial ratios: those made from a single-year financial statement, and those made from two-year financial statements.

1: Ratios generated from a single-year financial statement

This type of ratio is generated from one accounting period's financial data. We choose two arbitrary items from the balance sheet, profit-and-loss statements, and cash flow statements, including cases in which each item from a different financial statement is selected, and calculate the ratio. As replacing the numerator with the denominator generates an essentially identical ratio for bankruptcy prediction, we address either of them. An item with a value less than or equal to zero, for even a single company, will be handled so that it does not become the ratio's denominator. This type of ratio is represented as R-1 in subsequent sections.

\section{2: Ratios generated from two-year financial statements}

This type of ratio is generated from two successive fiscal periods' financial data. This ratio type is further categorized as three subtypes R-2a, R-2b, and R-2c, as follows. First, let us use $A(X)$ to represent the value of financial item Aof a certain company in year $X$.The denominator's value does not become o or less for all three types, as in the ratio R-1.

R-2a: This is defined as the ratio of a specific financial item $A_{1}$ between two successive years $X$ and $X-1$, representing $A_{1}(X) / A_{1}(X-1)$. By using R-2a, we expect to observe the changing rate of each financial item from the previous year.

R-2b: This is defined as the ratio of the sums of two financial items $A_{1}, A_{2}$ between two successive years $X$ and $X$-1, representing $\left\{A_{1}(X)+A_{1}(X-1)\right\} /\left\{A_{2}(X)+A_{2}(X-1)\right\}$. This summation plays a role of averaging, and we anticipate that this smoothing effect will enable us to grasp the change's outline more appropriately than R-1. 
R-2c: This is defined as the difference of a specific financial item $A_{1}$ between two years $X$ and $X-1$ divided by the value of another financial item of year $X$, representing $\left\{A_{1}(X)-A_{1}(X-1)\right\} /$ $A_{2}(X)$.We expect that this ratio can grasp the change of a financial item versus the value of another item.

\subsection{Financial ratio extraction and discrimination by AdaBoost algorithm}

It is difficult to derive an optimal combination of indicators from many generated financial ratios. We have overcome this difficulty by conducting the entire process, from selecting financial indicators to constructing a prediction model, in a single framework in AdaBoost. The algorithm in AdaBoost to select financial indicators (feature extraction) and derive a discriminant function will be explained below.

AdaBoost (Freund \& Schapire, 1997)was not originally designed for feature extraction. AdaBoost is a kind of ensemble learning algorithms which aim to create a single classifier with high accuracy, termed a "strong" classifier, by combining multiple classifiers with low accuracy termed "weak" classifiers. This study applies the algorithm to select financial indicators.

We first define the notation as it appears below. In defining the number of generated ratios and subject companies as $M$ and $N$, respectively, the financial ratio for each company is expressed as $\boldsymbol{x}_{i}(i=1,2, \ldots, N)$, and the class label as $y_{i} \in\{1,-1\}$. The $y_{i}=1$ in this article indicates a continuing company, while $y_{i}=-1$ notes a failed company.

The sequence of selecting financial indicators through AdaBoost is as follows:

1) Prepare a learning data set $\left(x_{i k}, y_{i}\right)(i=1,2, \ldots, N ; k=1,2, \ldots, M)$, where $x_{i k}$ represents the $k$-th element of a vector $x_{i}$.

2) Initialize weights $w_{i}(i=1,2, \ldots, N)$ to each sample (each company's data) equally:

$$
w_{i}=\frac{1}{N}(i=1,2, \ldots, N) \text {. }
$$

3) Prepare weak classifiers $h_{k}\left(x_{i k}\right)(k=1,2, \ldots, M)$ that correspond to a simple thresholding classifier with respect to the $k$-th financial ratio, which classifies the value of financial ratio $x_{i k}$ into either +1 (continuing companies) or -1 (failed companies). The optimal value of each classifier's threshold is derived beforehand.

4) Repeat the following procedures 5)-8) Ttimes.

5) Calculate the weighted error rates $\varepsilon_{t k}(t=1,2, \ldots, T ; k=1,2, \ldots, M)$ of each weak $\operatorname{classifier}_{k}\left(x_{i k}\right)$ under the current value of weights $\left\{w_{i}\right\}$ :

$$
\varepsilon_{t k}=\sum_{i: h_{k}\left(x_{i k}\right) \neq y_{i}} w_{i} \text {. }
$$

6) Select the best weak classifier $h_{k_{t}^{*}}$ that minimizes the weighted error rate among all the weak classifiers.

7) Calculate the selected weak classifier's reliability measure $\alpha_{t}(t=1,2, \ldots, T)$ using the error rate $\varepsilon_{t k_{t}^{*}}$ as

$$
\alpha_{t}=\ln \left(\frac{1-\varepsilon_{t k_{t}^{*}}}{\varepsilon_{t k_{t}^{*}}}\right)
$$

8)Update the weights $w_{i}$ so that the weights of companies misclassified by the selected weak classifier $h_{k_{t}^{*}}$ increase: 


$$
w_{i} \leftarrow \frac{w_{i} \exp \left\{-\alpha_{t} y_{i} h_{k_{t}^{*}}\left(x_{i k_{t}^{*}}\right)\right\}}{z_{t}}
$$

where the $Z_{t}$ is the normalization factor,defined as

$$
Z_{t}=\sum_{i=1}^{N} w_{i} \exp \left\{-\alpha_{t} y_{i} h_{k_{t}^{*}}\left(x_{i k_{t}^{*}}\right)\right\}
$$

By this, the weights of the samples that can be correctly categorized by the selected financial indicator (the $k_{t}^{*}$-th financial ratio) decrease, while the weights of the samples that are mistakenly categorized increase. Each sample weight is eventually normalized so that the sum of the weights becomes one.

By completing the aforementioned procedures, $T$ weak classifiers $h_{k_{t}^{*}}(t=1,2, \ldots, T)$ are obtained. With AdaBoost, the weights of the samples that are mistakenly categorized by the selected financial indicator increase, and in the later iteration step, financial indicators that can discriminate such "difficult" samples will become easier to select.

The AdaBoost algorithm providesa way to not only select financial indicators, but also predict the class label of new dataz $=\left(z_{1}, z_{2}, \ldots, z_{M}\right)$ corresponding to a certain company's financial ratios by the following formula (corresponding to a strong classifier):

$$
y_{z}=\operatorname{sign}\left[\sum_{t=1}^{T} \alpha_{t} h_{k_{t}^{*}}\left(z_{k_{t}^{*}}\right)\right] \text {, }
$$

where the function $\operatorname{sign}(\cdot)$ is defined as $\operatorname{sign}(x)=\left\{\begin{array}{l}+1(x \geq 0) \\ -1(x<0)\end{array}\right.$. The entire process is implemented within a common framework, from selecting financial indicators to constructing a prediction model, and an optimal set of financial indicators is naturally selected. This differentiates our proposed method from traditional ones that conduct both processes separately.

\section{Evaluation experiments and consideration}

This section will discuss the evaluation experiments.

\subsection{Experimental description}

We conduct seven experiments that are primarily categorized in two types: those that use financial data from one accounting period, and those that use financial data from two successive years. Table 1 illustrates the dataset utilized in each experiment.

Regarding the case of using a single-year financial statement (cases I, II, III, and IV), the data for both failed and continuing companies are obtained only for one accounting year. The ratios generated from a single-year financial statement (R-1 type) are utilized in these cases. This series of experiments aims to investigate the degree of performance deterioration, as the failed companies' data goes back from the date of their bankruptcy. It is noted that the more the utilized data goes back from the bankruptcy, the earlier the prediction is performed. Alternatively, in the case of using two years of financial statements (cases V, VI, and VII), the data for both failed and continuing companies are obtained for two successive accounting years. These cases utilize not only R-1type ratios, but also those generated using two years of data (R-2a, R-2b, and R-2c) as candidates for the financial indicators. These experiments aim to compare prediction performance with those that use a single year's data.

Table 2 indicates the total number of financial ratios generated using the procedure noted in Section 3.2. When financial data for one accounting period is utilized (cases I, II, III, and IV), the number of candidate ratios is always 546. On the other hand, the varied number of candidate ratios for cases V, VI, and VII is attributed to the fact that financial items that can be a denominator of ratio $\mathrm{R}-2 \mathrm{~b}$ differ depending on the years. 
Table 1: Dataset utilized in each case of our experiment

\begin{tabular}{|c|l|l|l|}
\hline Exp. & \multicolumn{1}{|c|}{ Failed Company Data } & \multicolumn{1}{|c|}{ Continuing Company Data } & \multicolumn{1}{c|}{ Used Ratio } \\
\hline I & within ONE year before bankruptcy & from July 2015 to June 2016 & R-1 \\
\hline II & within one year from TWO years before bankruptcy & from July 2014 to June 2015 & R-1 \\
\hline III & within one year from THREE years before bankruptcy & from July 2013 to June 2014 & R-1 \\
\hline IV & within one year from FOUR years before bankruptcy & from July 2012 to June 2013 & R-1 \\
\hline V & data of failed companies for I and II & data of continuing companies for I and II & $\begin{array}{l}\text { R-1, R-2a, } \\
\text { R-2b, R-2c }\end{array}$ \\
\hline VI & data offailed companies for II and III & data of continuing companies for II and III & $\begin{array}{l}\text { R-1, R-2a, } \\
\text { R-2b, R-2c }\end{array}$ \\
\hline VII & data of failed companies for III and IV & data of continuing companies for III and IV & $\begin{array}{l}\text { R-1, R-2a, } \\
\text { R-2b, R-2c }\end{array}$ \\
\hline
\end{tabular}

Table 2: Each experiment's number of candidate ratios

\begin{tabular}{|c|c|c|c|c|}
\hline Experimental Type & I, II, III, IV & V & VI & VII \\
\hline Number of Financial Ratios & 546 & 2,651 & 2,686 & 2,615 \\
\hline
\end{tabular}

In every experiment, while all 94 samples of failed companies are utilized, we randomly choose 94 continuing companies as samples from 2,287 complete datasets to strike a balance between the number of failed and continuing companies. Further, to alleviate the influence of this randomness, we run a 100-time implementation by changing the combination patterns of 94 continuing companies.

\subsection{Leave-one-out cross-validation}

Leave-one-out cross-validation is used in each trial to evaluate the proposed method. This method applies the following procedures: 1) financial indicators are selected with AdaBoost by using corporate data for 187 out of 188 companies as learning samples; and 2) assuming that the remaining one company data is a new one for the test, whose class label is unknown, this sample's discrimination is performed by using Equation(3.6). Changing this combination of learning samples and the test sample will allow for an examination of all 188 cases.

Eventually, there are a total of 18,800 predictions in each experiment. We define the identification rate as the proportion at which discrimination correctly occurs over all trials.

\subsection{Quantitative results of identification rates}

Figure 1 illustrates the discrimination accuracy obtained by the proposed method in each experiment. The horizontal axis represents the number of financial indicators considered in the prediction model, which equals the number of times AdaBoost repeats the process, namely, the value of $T$.The vertical axis indicates the identification rate averaged over all trials, through the continuing companies' recombination and the leave-one-out crossvalidation, namely, the number of correct discrimination divided by the number of entire trials 18,800 . 


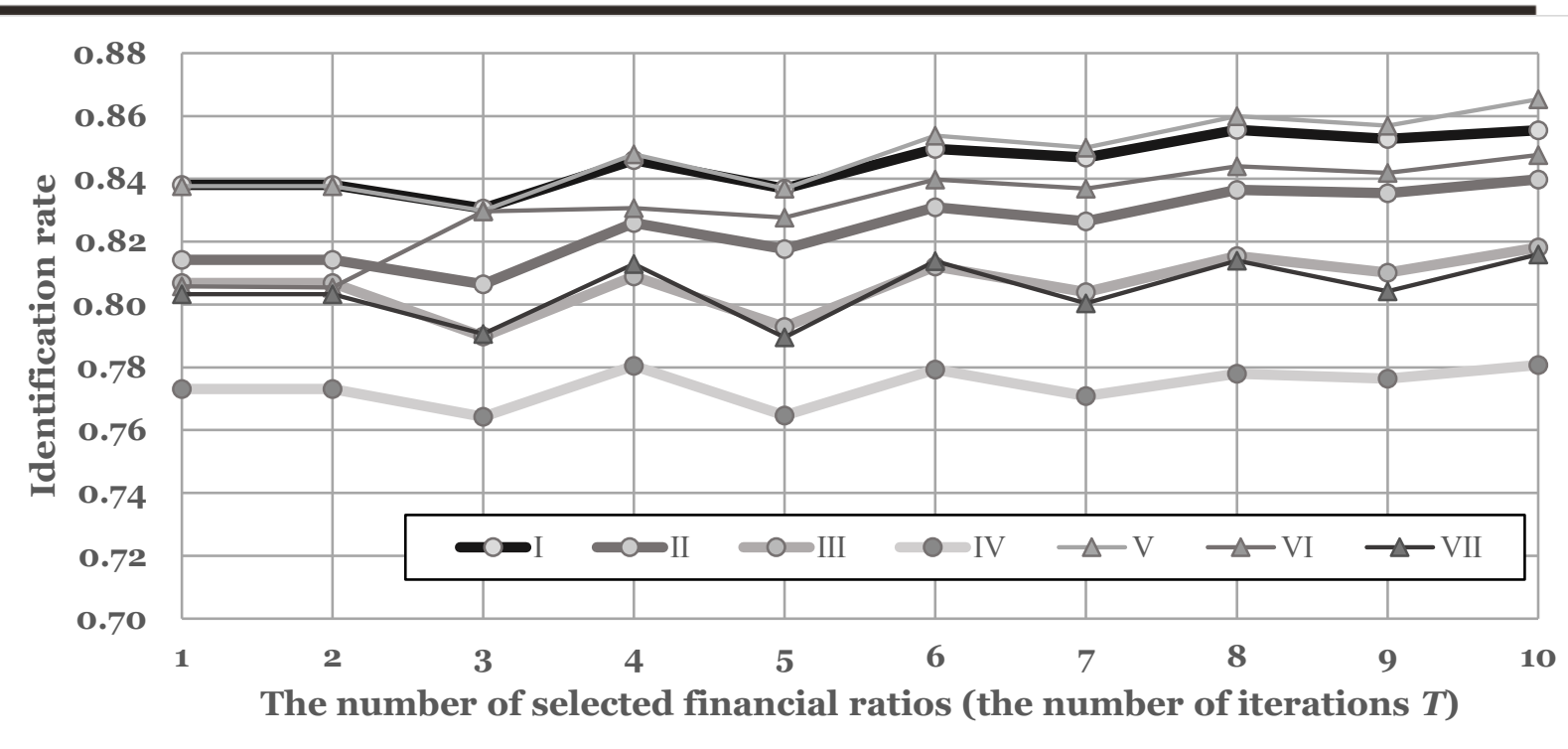

Figure 1. Precision of the proposed method relative to the number of selected financial ratios The best performance is achieved in the case of experiment $\mathrm{I}$, in which the data within one year before bankruptcy is utilized for the failed company. The identification performance in the single-year financial indicators (experiments I-IV) deteriorates as the failed companies' used data goes back from the bankruptcy date, which indicates the difficulty in the early prediction of business failures by using single-year financial data. It is further noted that the performance with financial ratios generated from multiple-year data (experiments V-VII)can be greater than when using only corresponding single-year financial ratios. This confirmed the proposed temporal financial ratios' effectiveness. Especially in the case that uses the data within one year from two years and three years before bankruptcy (experiment VI), the identification rate surpasses that of corresponding single-year cases (experiments II and III) after the third AdaBoost step. These results imply that our proposed method can predict oneyear future bankruptcies with high accuracy.

\subsection{Qualitative considerations for selected financial ratios}

We will next examine the selected financial ratios' qualitative validity from an accounting perspective.

Although the overall trend is that as the number of financial indicators increases, discrimination accuracy slightly improves, the first selected ratio seems highly critical. We examine the first three financial ratios picked up by AdaBoostfor the case that uses data within one year from two years and three years before bankruptcy (experiment VI). Table 3 illustrates the combination of the first three financial ratios that most frequently appear in the 18,800 trials.

These ratios are interpreted as follows:

(1) Retained earnings / Current assets (ratio type: R-1)

Failed companies tend to increase their current assets. This primarily seems to be because the collection of sales proceeds is unsuccessful and inventory increases. Additionally, the failed company's retained earnings are likely to decrease through financial difficulty. Therefore, this indicator seems to serve for discrimination between failed and continuing companies. 
(2) Valuation, translation adjustments, and others / Capital stock (ratio type: R-2c)

Valuation, translation adjustments, and others include unrealized gains on other securities and deferred hedge gains and losses. Many bankruptcy companies demonstrate values for this indicator of o or less. One possibility for this is that companies going bankrupt do not hold securities or they have bad debts. Capital stock in the denominator seemingly plays a role as a normalization factor reflecting the companies' scale.

(3) Loss on disposal, valuation of other assets / Capital stock (ratio type: R-2b)

Companies going bankrupt tend to dispose of fixed assets a few years prior to their actual bankruptcy; the numerator directly reflects this corporate behavior. Thus, the value of this ratio seems to highly differ between failed and continuing companies.

Table 3: The most frequently extracted combination of financial ratios in experiment VI

\begin{tabular}{|c|l|l|l|}
\hline Rank & \multicolumn{1}{|c|}{ Numerator } & \multicolumn{1}{|c|}{ Denominator } & \multicolumn{1}{|c|}{ Ratio Type } \\
\hline 1 & Retained earnings & Current assets & R-1 \\
\hline 2 & $\begin{array}{l}\text { Valuation, translation adjustments, and } \\
\text { others }\end{array}$ & Capital stock & R-2c \\
\hline 3 & Loss on disposal, valuation of other assets & Capital stock & R-2b \\
\hline
\end{tabular}

\section{Summary and future works}

This study has attempted early bankruptcy predictions from time-series financial data based on our past boosting approach to unify the selection of financial ratios and the construction of a prediction model. We have generated ratios from two arbitrary items in the balance sheets, profit-and-loss statements, and cash flow statements, and have evaluated the performance of the proposed method through the leave-one-out cross-validation. The experimental results indicate that combining financial data for multiple years can improve prediction accuracy. Specifically, in the case of using the data within one year from two years and three years before bankruptcy, an identification rate of $82.9 \%$ was achieved with three financial ratios, which implies that our method can predict one-year future bankruptcies. Our experiments indicate that the most helpful ratios for this early prediction were:

1) Retained earnings (present value) / Current assets (present value);

2) Valuation, translation adjustments, and others(difference between recent two years)/ Capital stock (present value); and

3) Loss on disposal, valuation of other assets(sum over recent two years) / Capital stock (sum over recent two years).

Future works could address some issues concerning stratified analyses. Although this study did not conduct an evaluation by industry, trends in financial indicators substantially differ from one industry to another in reality. Thus, an evaluation by industry may improve the prediction accuracy and may facilitate the discovery of more effective financial indicators. Further, as a business performance is influenced by external factors, including the economic environment, it is unlikely that specific financial ratios are always effective in predicting bankruptcies in any given period. Accordingly, the evaluation by year may lead to the discovery of new knowledge. 
Asia Pacific Journal of Advanced Business and Social Studies

ISBN (eBook): 9780994365675 | ISSN : 2205-6033

Year: 2017 , Volume: 3, Issue: 2

\section{Acknowledgement}

This research was supported by JSPS KAKENHI Grant Numbers 15K21395. 


\section{References}

i. Altman, E. I., Financial Ratios, Discriminant Analysis and the Prediction of Corporate Bankruptcy, The Journal of Finance, 23(4), pp. 589-609, 1968.

ii. Altman, E. I., G. Marco, and F. Varetto, Corporate Distress Diagnosis: Comparisons Using Linear Discriminant Analysis and Neural Networks (the Italian Experience), Journal of Banking \& Finance, 18, pp. 505-529, 1994.

iii. Beaver, W. H., Financial Ratios as Predictors of Failure, Journal of Accounting Research, 4, pp. 71-111, 1967.

iv. Edmister, R. O., An Empirical Test of Financial Ratio Analysis for Small Business Failure Prediction, Journal of Financial and Quantitative Analysis, 7(2), pp. 1477-1493, 1972.

v. Freund, Y., and R. E. Schapire, A Decision-Theoretic Generalization of On-Line Learning and an Application to Boosting, Journal of Computer and System Sciences, 55(1), pp. 119-139, 1997.

vi. Hosaka,T., and Y. Takata, Corporate Bankruptcy Forecast Using RealAdaBoost, iNFORMATION-An International Interdisciplinary Journal, 19(6B), pp.2285-2298, 2016.

vii. Ohlson, J. A., Financial Ratios and the Probabilistic Prediction of Bankruptcy, Journal of Accounting Research, 18(1), pp. 109-131, 1980.

viii. Ramakrishnan, S., M. Mirzaei, and M. Bekri, Corporate Default Prediction with AdaBoost and Bagging Classifiers, Journal Teknologi (Sciences \& Engineering), 73(2), pp. 45-50, 2015.

ix. Schapire, R. E., and Y. Singer, Improved Boosting Algorithms Using Confidence-rated Predictions, Machine Learning, 37(3), pp. 297-336, 1999.

x. Shin, K., T. S. Lee, and H. Kim, An Application of Support Vector Machines in Bankruptcy Prediction Model, Expert Systems with Applications, 28, pp. 127-135,2005.

xi. Shirata, Y., Formation of Bankruptcy Prediction Information (in Japanese), Chu-o Keizaisha, 1999.

xii. $\quad$ Shirata, Y., Bankruptcy Prediction Model (in Japanese), Chu-o Keizaisha, 2003.

xiii. Sung, T. K., N. Chang, and G. Lee, Dynamics of Modeling in Data Mining: Interpretive Approach to Bankruptcy Prediction, Journal of Management Information Systems, 16(1), pp. 63-85, 1999.

xiv. Takata, Y., T. Hosaka, and H. Ohnuma, Financial Ratios Extraction Using AdaBoost for Delisting Prediction, The Seventh International Conference on Information, Proceedings of the Seventh International Conference on Information, pp.158-161, 2015. 